\title{
Improving Operation Indices of a Micro-grid by Battery Energy Storage Using Multi Objective Cuckoo Search Algorithm
}

\author{
${ }^{1}$ Niroo Research Institute (NRI), Tehran, Iran \\ ${ }^{2}$ University of Mohaghegh Ardabili, Ardabil, Iran \\ ${ }^{3}$ Sarab Branch, Islamic Azad University, Sarab, Iran \\ ${ }^{4}$ Azarbaijan Regional Electric Company, Tabriz, Iran \\ ${ }^{*}$ Corresponding Author
}

S. Saeid Taheri ${ }^{1,2,3^{*}}$, Seyedjalal Seyedshenava ${ }^{2}$,Vahideh Mohadesi ${ }^{3}$, and Rasoul Esmaeilzadeh ${ }^{4}$

\begin{abstract}
In this paper, the effect of using Battery Energy Storage (BES) on the operation of power systems is properly investigated by implementing a multi-objective algorithm. In recent decades, the energy storage utilization has been taken into account for performance enhancement of the grid. Typically, there is a moment balance between the power production and consumption and no electrical energy storage therein. However, with the remarkable development of electrical storage technologies, many of the assumptions in electrical power storage systems are changing rapidly and a particular place for storage has been created in current power systems. Utilizing this equipment could cause economical and technical benefits in networks and each one of these issues should be analyzed seriously. In this paper, the potential effect of using BES on the improvement of some important indices of power system operation has been investigated like load factor, network losses and voltage index. So, an OPF model is proposed to find the optimal size and location of battery in the network to help the system operator in reaching its purposes. The integrated algorithm is used, and the efficiency and availability of the model has evaluated by implementing it within a model.
\end{abstract}

\section{Introduction}

According to the definition, an energy storage system is considered as a physical system with the ability to gain energy for dispatching electricity in next time periods. Currently, at least 140 GW big scale energy storage is installed in electricity networks, overall the world [1]. The most of these capacities include pump-hydro storage technology and a small part is dedicated to other technologies like batteries, compressed air saver, fly wheel, and hydrogen storage facilities. Nevertheless, the part of other technologies is changing with emerging the new technologies and also developing some of them. The most important reason of these changes is the researches done in the car making factories that strive to make cost effective electrical cars by lowering the price of battery. Nykvist in [2] states that the finished price of batteries is reducing to about 20 percent annually. The manufacturers and the electrical system managers are satisfied with the progresses done in the mentioned area and thinks that the battery technology will not be costly, in the future. So, the effective using study of the batteries for having the best performance in power systems is needed.

Batteries store the electrical energy via chemical interactions. In other words, charging a battery causes electro-chemical interactions of its elements and therefore saves energy chemically. On the basis of needs, the reverse chemical interaction causes flow of electric field from battery toward the network. One of the important features of some batteries is their rapid response; these batteries could meet the load variations at less than $20 \mathrm{msec}$. The efficacy of batteries is between 70 percent and 80 percent, which depends upon the type of battery and also the cycle of its usage. The stored energy losses of batteries are low and also they have high electrical energy storage density, in some cases. The cellular structure of batteries helps them to achieve the desirable capacity of power and voltage for the power system via interconnecting of battery cells. Various battery technologies that are presented in commercial markets can be divided to the main categories: Acid-lead, nickel-cadmium, sodium-sulfur, lithium batteries.

Received: March 23 $3^{\text {rd }}, 2020$. Accepted: March $14^{\text {th }}, 2021$

DOI: 10.15676/ijeei.2021.13.1.7 
Energy storage systems could include in different part of power system like generation, distribution, transmission side and finally in consumption part, all over the world $[3,4]$. The optimal place for utilization of storage technologies depends upon their capability and also the structure of power systems. In ref [5], a model is presented for computing the size of energy storage system in a micro-grid with considering the reliability. In this paper, the investment cost of storage facility and also the expected operation cost of the network are minimized and the notsupplied energy and energy lack, that caused by existing units turn-off or renewable units variations, is controlled via storage units. Therefore the reliability indexes of micro-grid are satisfied. It proposes a linear model and uses a mixed integer programming (MIP) method for solving the problem. Ahadi presents a method for optimal placement of solar and wind systems with energy storage by minimizing annual power losses [6]. The presented model is a probabilistic model for all normal conditions and use from mixed integer non-linear programming (MINLP) method for solving it. In ref [7], controlling of the distributed generations merged with storage systems is analyzed for compensation of voltage drop in distribution system. Considering the importance of distributed generation unit control, the combination control of fuel cells and energy storage is presented and is analyzed when voltage dropping event occurred. Reference [8] considers the modeling and the simulation of old and new (like battery) type of energy storages in smart grids. In this reference, the specifications of different system controllers are presented and the advantages and disadvantages of battery energy storage system will be analyzed, too. In a similar way, Nakayama in [9] considered the compressed air energy storage. But in reference [10], a new insight for improvement of the voltage profile is presented in distribution systems in presence of distributed generation resources and energy storage systems. This reference analyzes the accompaniment of society members and PV variety and the installed capacity and then determines the storage system capacity. The other reference [11] presents that the application of storage facilities is increasing and it could help for the distribution systems for transferring from regular networks towards smart-grids and this could be considered as one of the best advantages of these technologies. Ref. [12] has strived to help operator and wind farm owners in making optimal decisions about expanding and improvement of electrical system performance by proposing a new model. This model determines the size of battery storage for electrical systems including wind farms in such a way that the value and cost of system reliability will be optimized. The same work is done in [13], considering the fuel and pollution costs of thermal units. In addition to the mentioned models, the optimal operation of storage facilities alongside the wind energy is considered in [14]. Also, ref. [15] tried to propose a method for planning and operation of wind turbines and energy storage in a restructured environment. The mentioned model uses energy storage for smoothing the wind unit production fluctuations, in order to help them to carry out their commitments in the market. This article shows that the micro-grid connection costs to the overall network could be reduced in case of enhancing the network with the energy storages.

The peak reduction could be considered as one of the main problems of generation and transmission of electrical power systems. Also, it is an important concern for electrical distribution companies for providing of the required power in the peak hours of the load. There are different methods for peak load reduction in power systems. One of these ways is the utilization of energy storage systems as a sub-structure for development and enhancement of smart grids. In ref. [16], a new method is presented for determining the size and optimal operation of BES that cause to the peak reduction, operational cost minimization and decreasing the overall cost paid by the customers, at the end. Besides that, the new energy resources have a main role and have employed extendedly in smart grids for supplying electrical power. Due to the stochastic nature of some resources, for example wind and sun, the predicting of the output power is difficult and this causes to the severe oscillations in the output power and brings different problems for the power system performance. Therefore, the utilization of energy storage systems in different parts of power system is necessary to maintain the power balance between the production and the consumption using energy storage at time periods which the production is less than the consumption. There are different models for improving of power system indices 
using the different search algorithms; but the objective function that presented in these models either solved the problem by a single objective that may involve different objectives with their related factors $[16,17]$. Therefore, considering the mentioned subjects here, the optimal operation of battery storages as a multi-objective problem did not investigated enough, considering losses, load factor and voltage profile, but it has been mentioned in this paper by multi-objective algorithm for studying its capability in solving these type of problems.

In this paper a multi-objective model is proposed for the peak reduction problem considering voltage stability index, load factor and the system losses using the multi-objective cuckoo search algorithm. The cuckoo search algorithm (CSA) is a search algorithm that shows its capabilities in solving different problems. For instance, ref. [17, 18] use this algorithm for solving of investment problems and ref. $[19,20]$ used this algorithm for implementing the optimal operation in micro-grids. In the second part of the paper, the specifications of CSA will be explained in short. The third part, explains the MO-CSA and the related flowchart of problem will be presented. Then in the fourth part, the applicability of the proposed model will be analyzed by implementing it on a power system and the related results will be presented.

\section{Problem Modeling and Assumptions}

If a power system security went to be low, the big industries will go towards the utilization of high capacity energy storages. The energy storages could help to the spinning reserve and also could reduce the load peak as their main function. Considering the peak time consumption price, the peak reduction capability is interested for the industries, which could cause to their payment reduction and make the battery storage investment more cost effective.

Sometimes the network loads includes intermittent consumptions at peak times that could cause high prices imposing into the system. This problem exists for many years for the electricity industries and they usually wanted to solve the problem via reduction of the peak load consumption. But today the battery energy storages are able to inject the electricity in short period of times and peak times. As it presented in figure 1, they could store energy when the price of the energy is low (for example at night) and reduce the peak time load with expensive price.
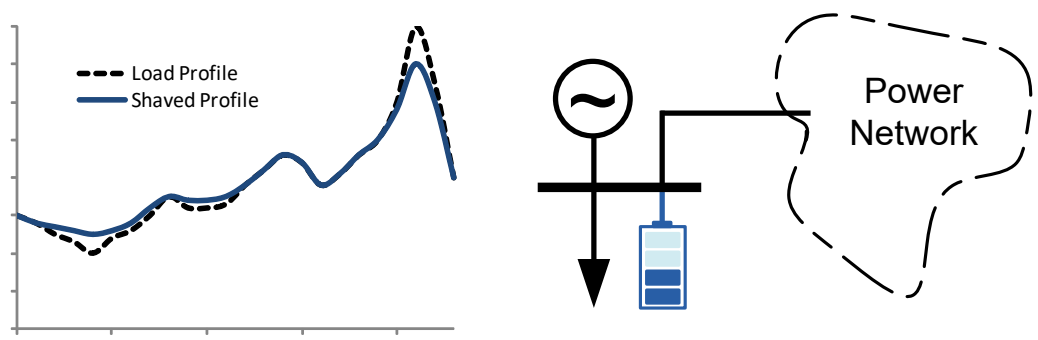

Figure 1. Peak shaving by Battery Energy Storage

This paper aims to improve the considered indices of the network operator using a multiobjective search algorithm. These parameters include the load factor, network losses and the voltage index of the system that have been formulized and presented as a multi-objective problem [21]. In this model, the investment cost of the battery storage is not counted and merely the networks parameters improve are considered, from the network operator point of view. The constraints which these targets can be achieved based on their, are the network power flow relation, and the limitations of energy storage units.

The objective function formulations can be presented as below:

- The load factor improvement 


$$
F_{1}=L F=\frac{\text { average }\left\{\sum_{n=1}^{N} P d_{n 1}, \sum_{n=1}^{N} P d_{n 2}, \ldots, \sum_{n=1}^{N} P d_{n 24}\right\}}{\max \left\{\sum_{n=1}^{N} P d_{n 1}, \sum_{n=1}^{N} P d_{n 2}, \ldots, \sum_{n=1}^{N} P d_{n 24}\right\}}
$$

- Minimizing the network losses

$$
F_{2}=P_{\text {Loss }}=\sum_{t=1}^{24} \sum_{l=1}^{L} \operatorname{real}\left(V_{n t}\left(\frac{V_{n t}-V_{m t}}{Z_{n m}}\right)^{*}-V_{m t}\left(\frac{V_{n t}-V_{m t}}{Z_{n m}}\right)^{*}\right)
$$

- The voltage profile index minimizing

$$
F_{3}=V I=\sum_{t=1}^{24} \sum_{n=1}^{N}\left(V_{\text {rated }}-V_{n t}\right)^{2}
$$

$\mathrm{N}$ is the bus numbers of the network, and $\mathrm{L}$ is the number of network lines. $P d_{n t}$ is the amount of consumed power in bus $\mathrm{n}$ and at the time of $\mathrm{t}$, and $V_{n t}$ is the amount of voltage in bus n and time t, and finally $V_{\text {rated }}$ is the amount of nominal voltage of regulated voltage in bus n. also, $Z_{n m}$ is the impedance of the line $\mathrm{n}-\mathrm{m}$.

Also, the conditions related to the power balance in each bus, and the minimum and maximum allowed voltage of each bus, the production limits of generation units and the restriction for power lines must be modeled as below:

$$
\begin{aligned}
& P g_{n t}+\left(P_{t}^{E}\right)_{n \in \Phi_{E}}-P d_{n t}-V_{m t} V_{n t} Y_{n m} \cos \left(\delta_{n t}-\delta_{m t}+\theta_{n m}\right)=0 \\
& V_{\text {min }}^{n} \leq V_{n t} \leq V_{\max }^{n} \\
& P g_{\min }^{n} \leq P g_{n t} \leq P g_{\max }^{n} \\
& S_{n m}=V_{m t} V_{n t} Y_{n m} \cos \left(\delta_{n t}-\delta_{m t}+\theta_{n m}\right) \leq \mathrm{S}_{n m}^{\max }
\end{aligned}
$$

The electrical storage sources (ESS) like battery banks are a certain dispatch units that are able to store (absorb) and inject the electrical energy. But like other electrical equipments, the battery storage has its own limitations [22]. On the basis of relation (8), the injected power of battery must be less than the maximum allowed dispatched power in every hour. Also the time continuity and the amount of stored energy at the after and before hour of each time period should be considered using the conditions (9) and (10). Finally, we must note that according to (11), the amount of restorable energy in battery is limited too and is less than a maximum amount.

$$
\begin{aligned}
& \left|P_{t}^{E}\right| \leq R P_{\max }^{E} \\
& \operatorname{Cap}(t+1)=\operatorname{Cap}(t)+P_{t}^{E} \\
& \operatorname{Cap}(0)=C_{S}, \operatorname{Cap}(T)=C_{E} \\
& \max \{\operatorname{Cap}(t)\} \leq \operatorname{Cap}^{\max }
\end{aligned}
$$

In relations (8)-(11), the $\mathrm{P}_{\mathrm{t}}^{\mathrm{E}}$ is the output power at hour $\mathrm{t}, \mathrm{RP}_{\max }^{\mathrm{E}}$ is the limitation of the maximum allowable flow rate of charge and de-charge. $\operatorname{Cap}(\mathrm{t})$ is the amount of stored energy in battery until hour $\mathrm{t}$. Also, $\mathrm{C}_{\mathrm{S}}$ and $\mathrm{C}_{\mathrm{E}}$ are the amount of energy at the initial and final time block. Finally, $\mathrm{Cap}^{\max }$ is the maximum restorable energy in ESS. 


\section{Multi-objective Cuckoo Search Algorithm}

The optimization techniques are applicable in a range of engineering issues, including engineering of power systems. Generally, the methods of solving optimization problems are divided into two main categories; the first is belongs to the classical methods such as linear programming, dynamic programming, mixed integer programming and other related techniques. These methods have limitations in solving nonlinear problems, which make it difficult to use these methods in real problems, which are mostly nonlinear [23, 24]. The second group of these methods includes intelligent methods that have found extensive application in engineering issues, nowadays. With regard to the nonlinear nature of real optimization issues, the mentioned methods could be very applicable. The accuracy of finding the optimal answer and the problem solving time, are important in solving optimization problems that the heuristic algorithms have advantages in short solving time. Among intelligent algorithms, nature-based algorithms are more prevalent than the other intelligent techniques

The optimization techniques explore the search space of optimization problems in different ways. The classical methods of optimization have been employed for long time but the applicability of these methods is limited for real-world problem owing to nonlinear constraints. However, the performance of meta-heuristic methods has been validated by real applications including different types of constraints. Among these methods, the nature inspired optimization algorithms have been extensively investigated during the last decade.

CSA is a new heuristic method that works based on breeding behavior and the characteristics of cuckoos in reaching best habitat societies of cuckoo. This method has been developed by Yang and Deb in 2009 [25]. It is a population-based search procedure to be used as an optimization tool for solving complex, nonlinear, and non-convex optimization problems. It has been shown that it is a promising algorithm which outperforms existing algorithms such as PSO and GA [26]. The follows explain the method in detail.

\section{A. Cuckoo Breeding Behavior}

CSA has been developed based on parasitic breeding behavior of some species of cuckoo. Quite a number of species engage in the obligate brood parasitism by laying their eggs in the nests of other host birds (often other species). Some parasite cuckoo species lay eggs that appear to mimic the appearance of the eggs of their favorite hosts hindering discrimination and removal of their eggs by host species. This cuckoo's feature increases their fertility by reducing the probability of their eggs being discovered by the host bird. It is obvious that if the host birds detect the intruders, they will throw alien eggs away. This behavior called as nest takeover. On the other hand, less aggressive hosts will simply abandon its nest and build a new nest in another location. The results are the same in both cases and finally the parasite cuckoo egg is ruined. The cuckoo usually takes its egg to a nest that the host birds' egg is there. In general, cuckoo's eggs hatch earlier than their host eggs. As soon as the cuckoo chicks have hatched, they lift any other eggs they find in the nest and then throw them overboard. Hatching early means that cuckoo chicks can oust other birds' eggs so that they can get more portion of the food from their foster parents. Studies also show that a cuckoo chick can also mimic the call of host chicks to gain access to more feeding opportunity.

\section{B. Cuckoo Search Implementation}

In this section, the breeding behavior of cuckoos and the characteristics of cuckoos in reaching best habitat societies are employed to reach a solution for optimization problems [2729]. It is concluded that the random walk via Lévy flight is much more efficient in exploring the search space as its step length is quite long in the long run of this algorithm. In order to simplify the description of novel CSA algorithm, three idealized rules can be used [25]:

1. Only one egg at a time is laid by cuckoo (each nest could involve only one solution in it),

2. Cuckoo dumps its egg in a randomly chosen nest,

3. Only the best nests with high quality eggs (best solutions) will be passed into the next generation. 
4. The number of available host nests is fixed.

5. Egg laid by a cuckoo bird is discovered by the host bird with a probability "pa". In this case, the host bird has two options. It can either throw the egg away or it may abandon the nest and build a brand new nest at nearby location.

6. The discovered nests in the previous step should be replaced by new random nests in other locations (new random solutions)

\section{Lévy Flight}

Many studies have shown that the flight pattern of many animals and insects demonstrates the typical characteristics of Lévy flights $[30,31]$. Such behavior has been applied to optimization algorithms for optimal search. Preliminary results show its promising capability in search the solution area with acceptable approximation so it is added to the CSA to improve its performance. CSA uses the Lévy flight in generating new solutions $x i(t+1)$ for the cuckoo number $i$ according to Equation (15).

$$
\mathrm{x}_{\mathrm{i}}(\mathrm{t}+1)=\mathrm{x}_{\mathrm{i}}(\mathrm{t})+\alpha^{*} \text { Lévy }(\lambda)
$$

where $\alpha$ represents the movement step and should be related to the scale of optimization problem. Lévy $(\lambda)$ is a number based on Lévy distribution.

\section{Multi Objective Solving Method}

In this section, the optimization model is a problem of selecting the appropriate storage size in a power system by the MO-CSA. Optimization is actually finding feasible answer or responses of the problem among feasible choices (subject to the constraints of the problem) and in order to optimize the target or the aims of an issue. Multi-objective optimization is a subset of a set of Multi Criteria Decision Making methods (MCDMs) that is made up of an unlimited set of feasible responses $[32,33]$. In these problems, unlike the one-goal problems, a set of answers is obtained. The overall form of these issues is as follows:

$$
\begin{aligned}
& \min f(x)=\left\{f_{1}(x), \ldots, f_{n}(x)\right\} \\
& \text { s.t: } \\
& g(x) \leq 0 \\
& h(x)=0
\end{aligned}
$$

By finding each new answer for this issue, it should be found whether or not it is dominated. If $A$ and $B$ are two different vectors for the answers of a multi-objective problem as $A=\left[a_{1}, a_{2}\right.$, $\left.\ldots, a_{k}\right]$ and $B=\left[b_{1}, b_{2}, \ldots, b_{k}\right]$, the definition of dominance will be the one in which the answer $A$ overcomes the response of $\mathrm{B}$, if it is not worse in any objective, and at least one of the objectives is better than the other one [34]. In other words, no component of A is larger than the corresponding component of B, and at least one component is smaller. Such a definition can be expressed mathematically, in the following order:

$$
\forall i \in(1, \ldots, k), a_{i} \leq b_{i} \wedge \exists i \in(1, \ldots, k): a_{i}<b_{i}
$$

If the new answer B satisfies such conditions, this answer is a dominated response and it will be deleted from the set of answers; otherwise the non-dominated response will be added as a new response to the existing Pareto response set. The Pareto front (PF) of a multi-objective problem can be defined as the set of non-dominated solutions. In-fact, the goal of the multi-objective optimization problem solving is to find its Pareto answers.

After finding the Pareto optimal solutions using MO-CSA, the best compromise solution should be chosen to demonstrate proper technique. In other words, an appropriate response must be selected as the final answer to determine a specific strategy for the problem. The final answer is called the best compromise solution that is a non-dominated answer according to the specific preference of different issues. Goal attainment method is a reliable and powerful optimization technique for finding the best compromise solution and near global optimization among Pareto set in multi-objective optimization problems. This method can be used in non-convex as well as convex problems [35]. In this paper, Goal Attainment method is employed for solving the multiobjective BES placement problem as well as other power system problems that using this method 
[36-38]. The specific details of this technique have been adequately discussed in many published articles and could be handled through these references [39, 40].

Similar to the single objective CSA, the main problem solving steps for MO-CSA can be summarized as the pseudo code shown below [41]:

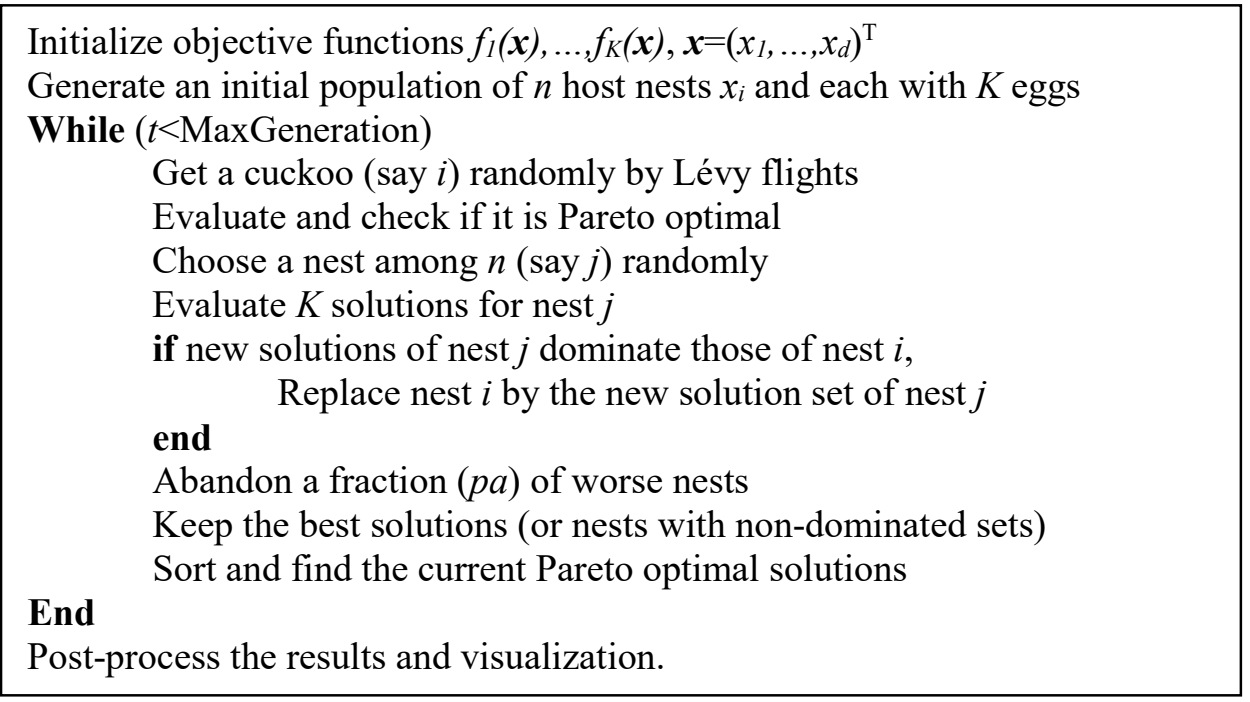

Figure 2. Multi-Objective Cuckoo Search Algorithm pseudo code

The independent variables of optimal storage sizing problem include battery storage charging and discharging amount during a day and the location and size of it. Meanwhile, the variable that belongs to the location is a discrete variable and the remaining variables are continuous. So if we want to express each nest for the algorithm, it could be shown as $\mathrm{Xi}=[\mathrm{Ni} \mathrm{Ci} \mathrm{P1i} \mathrm{...} \mathrm{P24i],}$ which contains the bus number $(\mathrm{Ni})$, the installed battery storage capacity $(\mathrm{Ci})$ and the charge or discharge variable as Pti. Index $i$ represents the number of nests that is considered in the algorithm. It should be noted that all of the variables use uniform probability distribution for generating random quantities. According to the recent points and considering problem constraints, the initial population is generated. After producing the initial population, the nest of each cuckoo is evaluated by objective functions and sorted according to the domination definition.

The proper numbers of nests could be about 20 in [42], but if the optimization problem were complex and has multiple numbers of local optimum points, then the number of nests could be more. In order to generate more optimal points on the Pareto front, we can increase the population size $n$. Through simulations, we found that 50 nest could be acceptable that increase a little computing time. The movement step in Lévy Flight is considered to be equal to 1.5. The proper repetition number for achieving optimum response is about 200 . However, the repetition number is adjusted to 500 for more reliability. In all simulations, the discovering probability (pa) is considered to be 0.25 .

Based on the above-mentioned rules, the CSA can be represented by the flowchart of Fig. 3 $[43,44]$. This algorithm is used to search for a multi-objective problem, and ultimately the most important response is presented as the final answer. In order to evaluate the efficiency of the proposed algorithm, the model presented in the following part of the paper will be applied to a numerical system. 


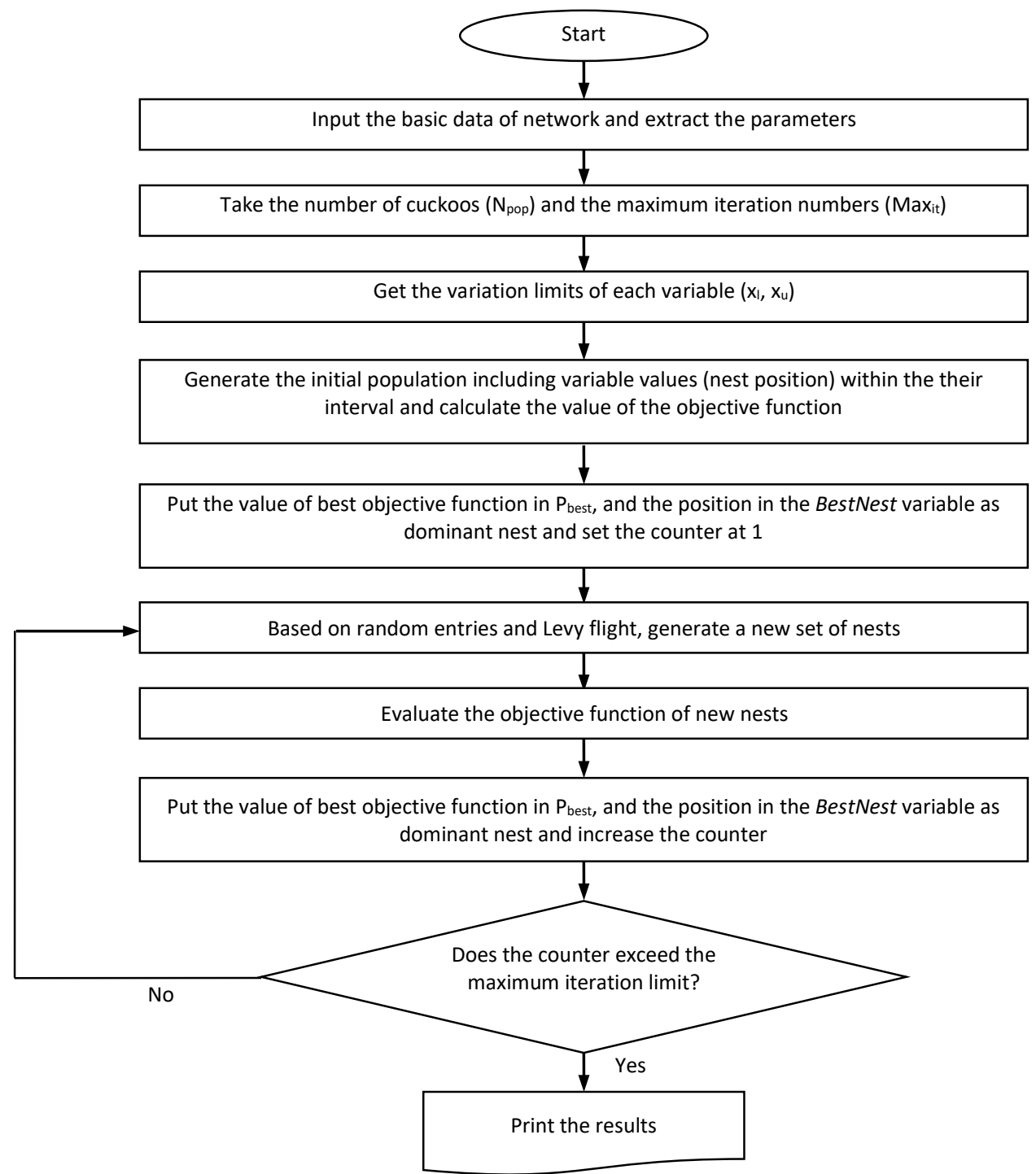

Figure 3. The flowchart of Multi Objective Cuckoo Search Algorithm (MO-CSA)

\section{Numerical Studies}

In this paper, the 24-bus IEEE RTS network is used for analyzing the proposed model [45]. This network has 10 generator, 34 transmission lines and 20 loads on its busses. The input information of this paper including the network characteristics information is presented in [45]. The chosen storage for this paper is a battery with the maximum capacity of $1800 \mathrm{MWh}$ that has a maximum transmission power of 300 megawatt in an hour. The network hourly load profile is shown in figure (4) and the amount of each load is counted with the same factor in different hours [46] at each bus. In this research, four study cases will be discussed for showing the model capability:

- In the first case study, the aim is to improve every three purpose including the load factor increasing, loss reduction and the voltage stability index reduction.

- In the second case study, the objectives are the load factor increasing and also the voltage index reduction in the network.

- In the third case study, the objective functions includes the network load factor increasing and of network loss reduction. 
- In the fourth case study, it is tried to improve all the first case purposes with a new load profile.

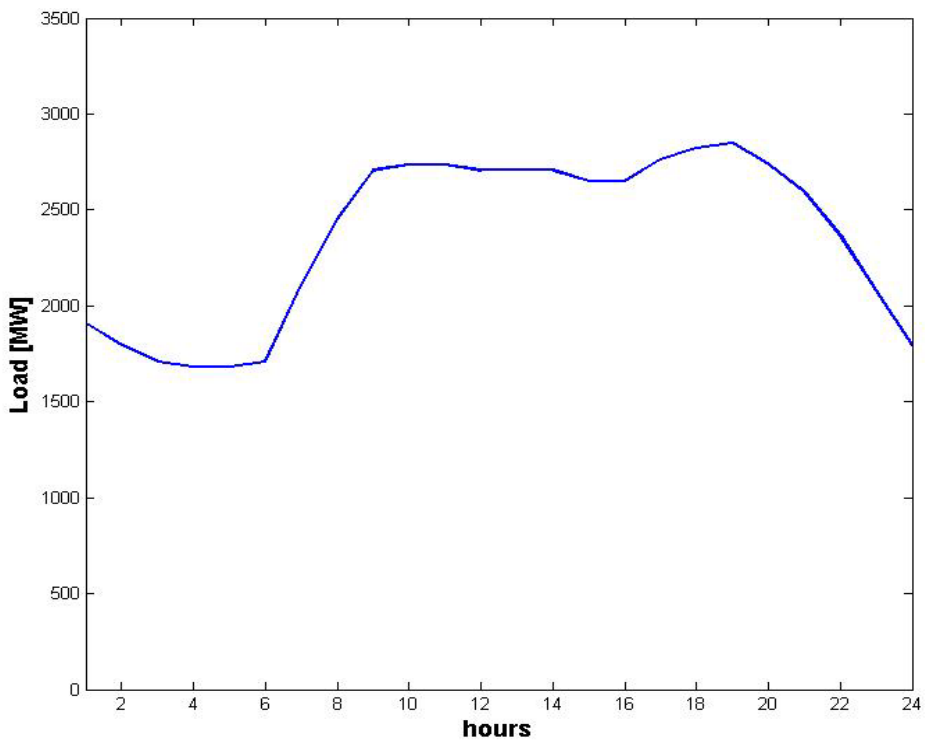

Figure 4. Initial load curve of 24-bus IEEE grid

In the first case study, all determined purposes in the second part of the paper are selected as multi-objective purposes of MO-CSA. In this case, the amount of load factor increased from 82.83 into 88.11 and also, the amount of network losses reduced from 1707.6 to $1664.81 \mathrm{KW}$. For gaining this purpose, the proposed algorithm installed battery storage with the capacity of $1800 \mathrm{MWh}$ on bus 10. The operation schedule of proposed storage is shown in figure 5 . Considering the operation schedule of storage, the algorithm wants to smooth the load curve with storing the energy during the base load hours of the day and injecting it at peak hours. Further, the load profile, before and after the storage installation, is presented in figure 5.

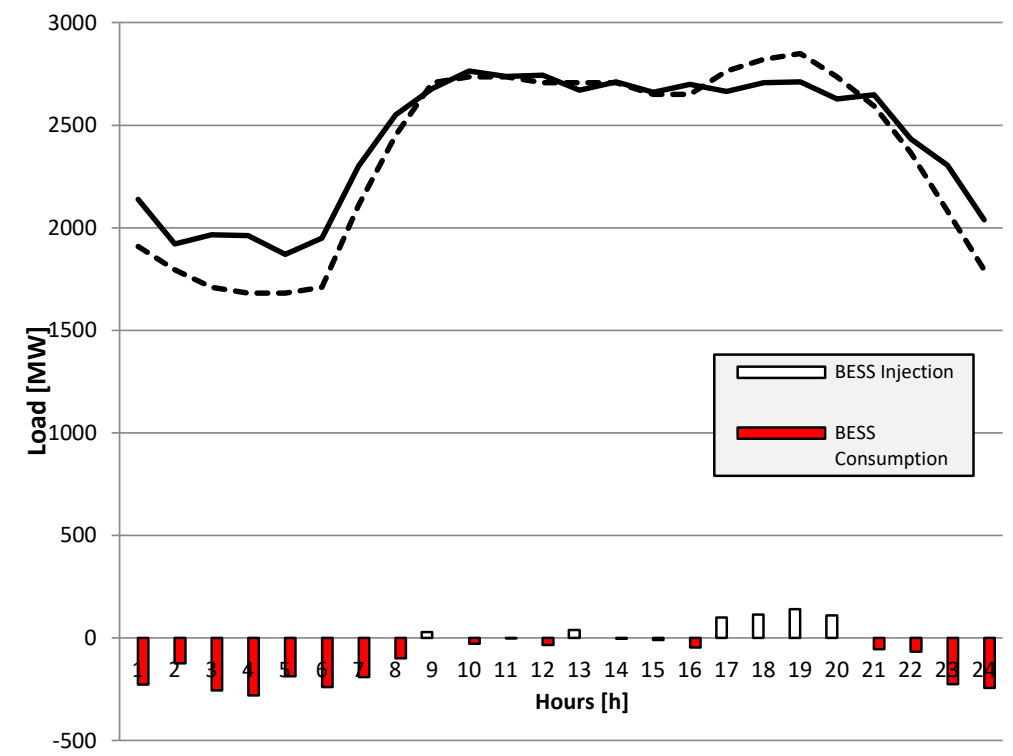

Figure 5. The operating charge/discharge graph of battery storage in IEEE 24-bus grid for case 1 
Considering the voltage index, the model presents good results and increased it from 0.1536 to 0.1416 . For evaluation of the energy storage installation effect on the network voltage profile, it has plotted in figure 6 for the maximum load of the network (peak load). The curves demonstrate that the voltage magnitude are decreased in some busses and are increased in other ones. This plot shows that the voltage magnitude in the peak hours is increased in bus 10 , that the storage is installed on it. This effect is seen in the busses that are near to bus 10, but when we get far from the considered bus, the voltage improvement effect is reduced.

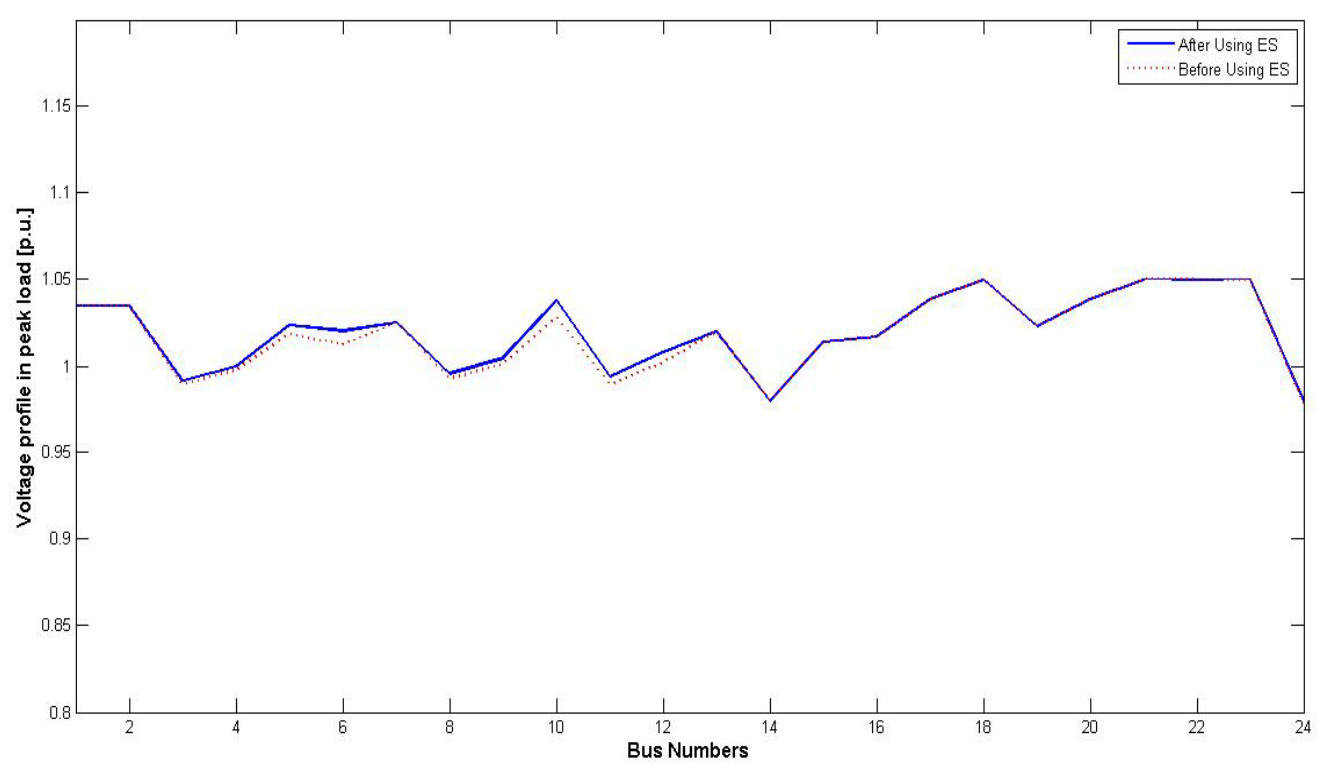

Figure 6. The peak hour voltage profile of IEEE 24-bus network for case 1

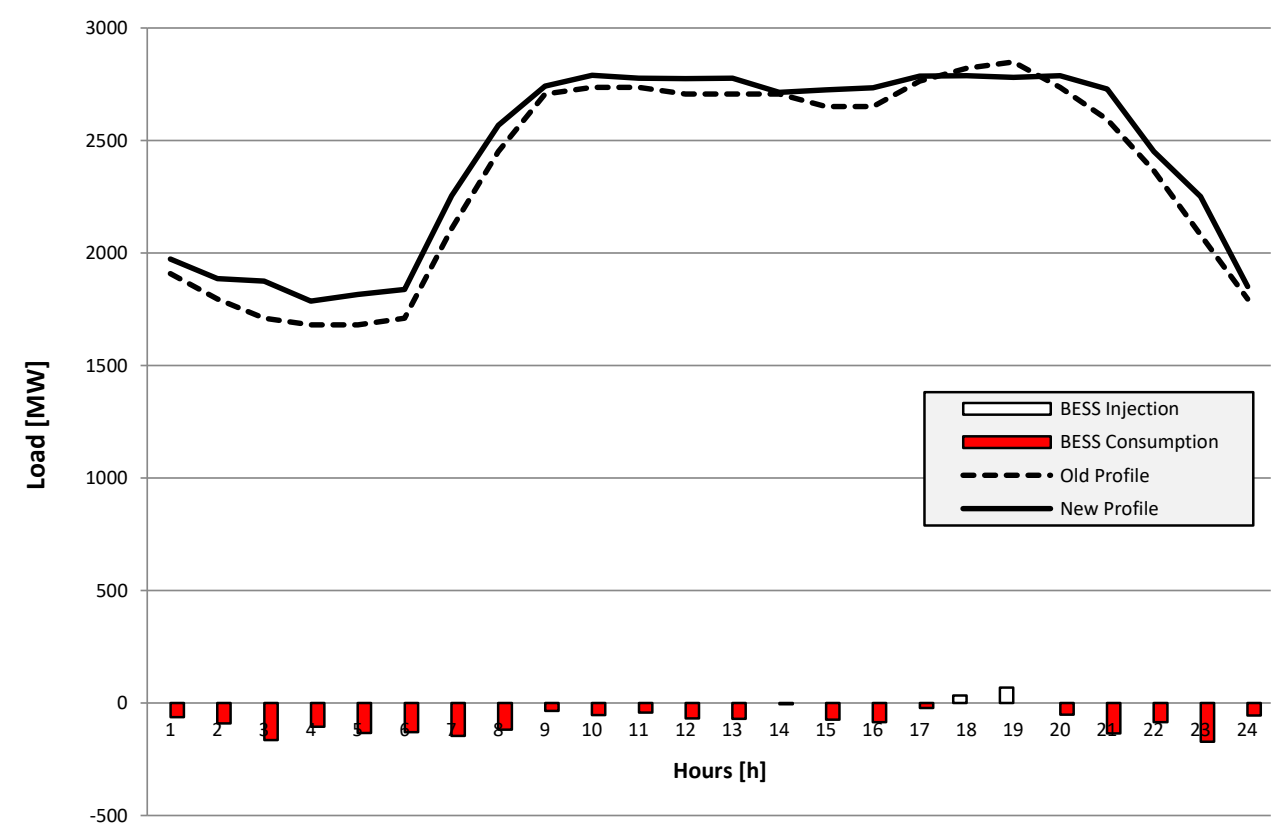

Figure 7. The operating charge/discharge graph of battery storage in IEEE 24-bus grid for case 2 
In the second case study, the load factor increasing and voltage index reduction of the system are the main objectives. In this case, the amount of load factor is increased from 82.83 to 87.33 that shows the proper operation of storage facilities during 24 hour that could be illustrated in figure 7. Also, the load profile of the network before and after operation of storage facility is shown in the mentioned figure, too. The figure presents that the load profile curve were smoother with storage facility. The figure shows that the storage sends less energy than what it has saved, to the network for improving the load factor. For this purpose, one battery with the size of 1800 MWh is proposed by the algorithm on bus 6 .

In this case, the loss reduction objective did not considered in the solutions and so, the amount of network loss increased to $1738.68 \mathrm{~kW}$. According to the operation schedule of the battery storage, the facility injects the most of its stored energy in the peak hours for improvement of the load factor beside the network voltage drop reduction. In this case, the amount of voltage index is decreased from the basic amount of 0.1536 to the 0.1349 and improved considerably with respect to the first case study in which the amount of losses is also chosen as the main objective. Figure 8, shows the voltage profile of the network before and after installation of the battery for peak hours. This figure shows that the amount of voltage in $n_{6}$, which the storage is installed on it, is improved in the peak load.

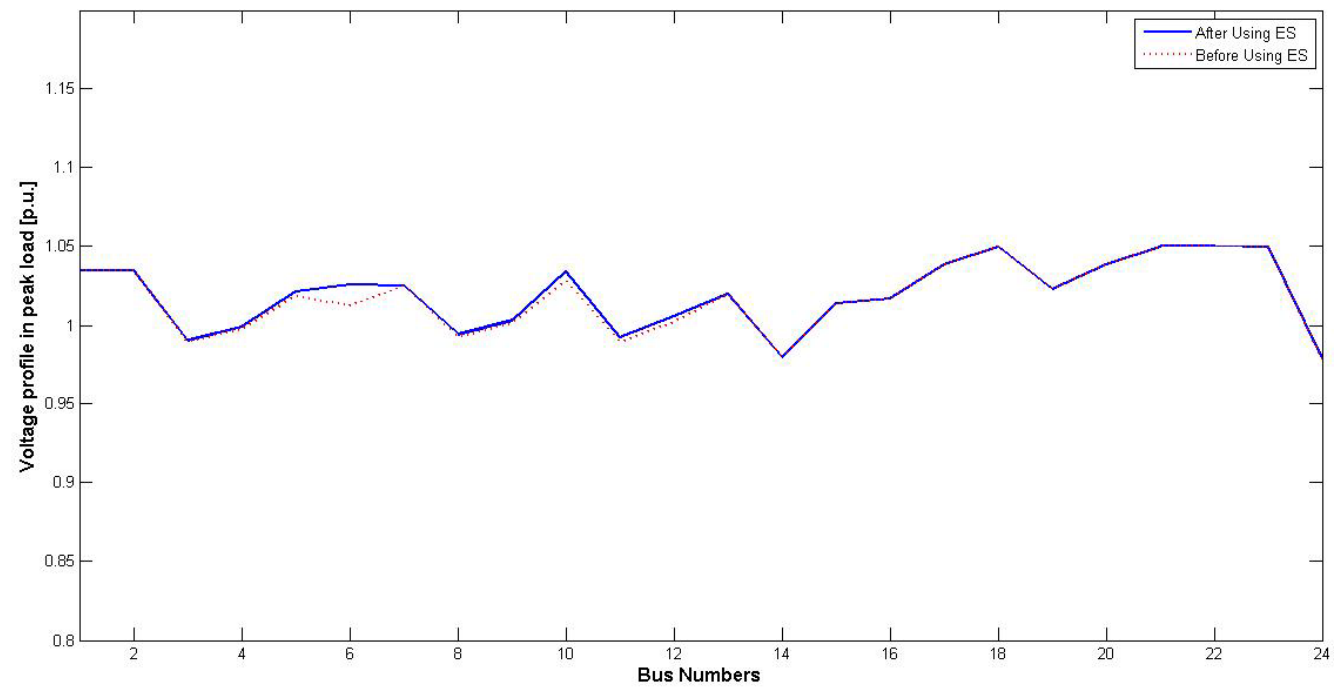

Figure 8 . The peak hour voltage profile of IEEE 24-bus network for case 2

The loss reduction and the load factor correction is the two main objective of the third case study. In this case, the amount of load factor is increased to 88.39 percent and the system loss that is the second objective of this case study, is decreased into $1434.84 \mathrm{~kW}$. So, the algorithm proposed an 1800MWh battery on bus 22 and recommends an operation schedule as presented in figure 9. The daily load profile of the network before and after installation of the battery storage is shown in the same figure, too. 


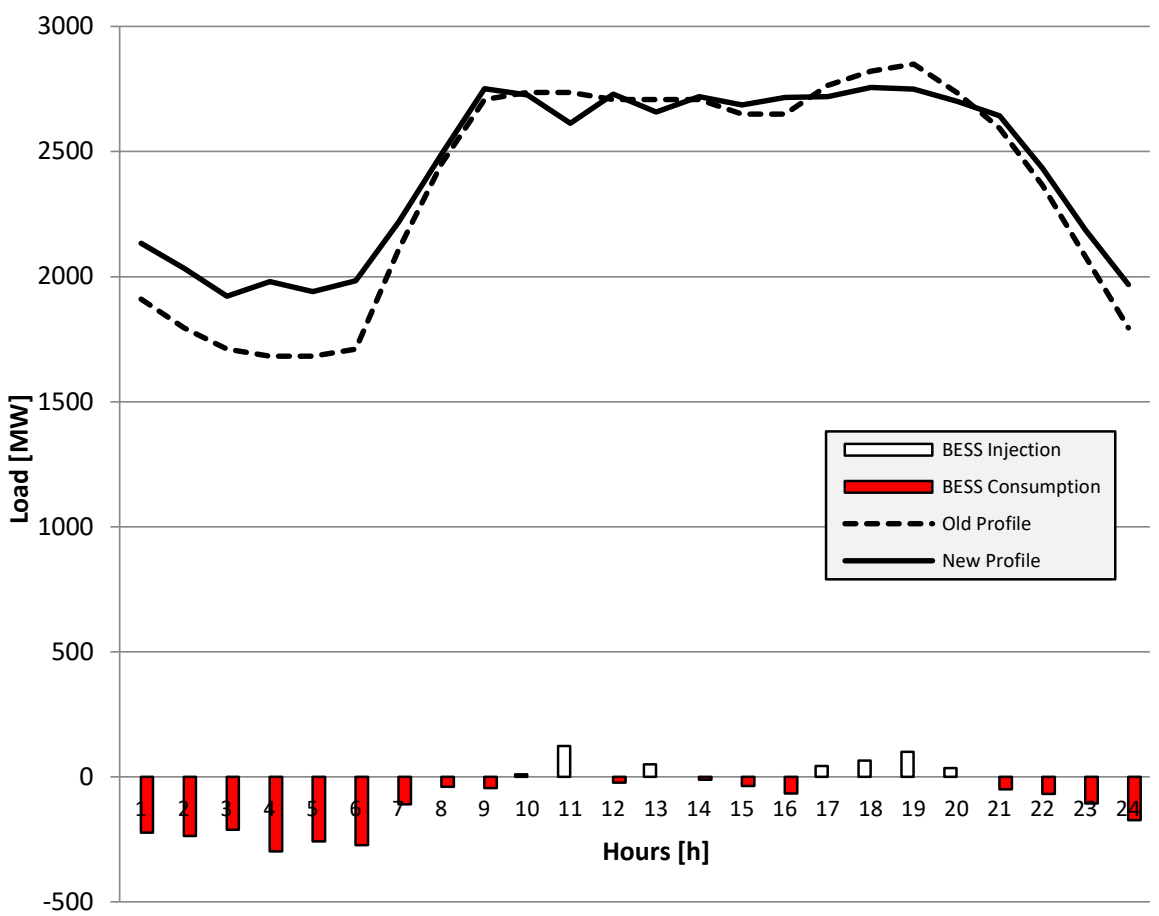

Figure 9. The operating charge/discharge graph of battery storage in IEEE 24-bus grid for case 3

The voltage profile index improvement is not the case in this section; so, the amount of this index went to be worse in this case study. The amount of voltage stability index has reached 0.1643 in this case study, and the magnitude of bus voltages is having more differences from the normal value. Figure 10, shows the network voltage profile before and after battery operation during the maximum peak load hour.

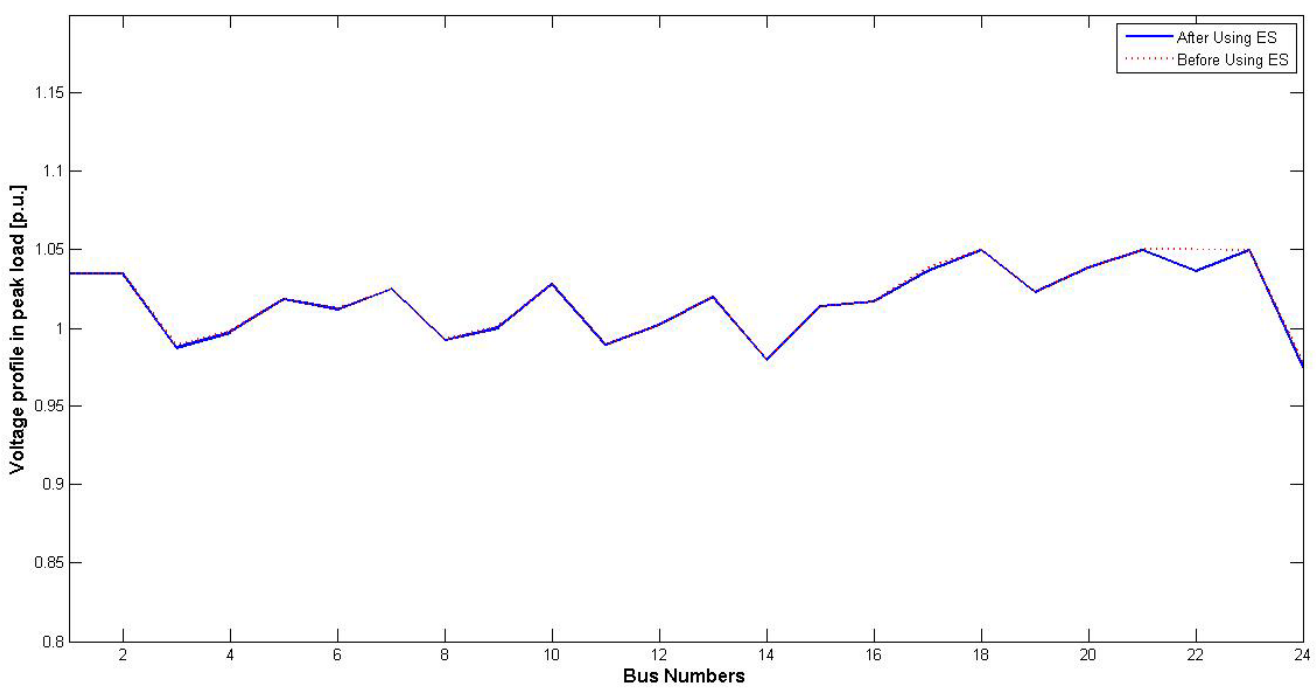

Figure 10. The peak hour voltage profile of IEEE 24-bus network for case 3 
The last case study (case 4) is studying the effect of the initial load factor on the final results of the problem. For this propose, the daily load curve shape is changed and the output results are analyzed. The new daily load curve is shown in figure 11. It is obvious that the load factor of this curve is lower and it has a sharp rate for the load growth comparing to the previous one.

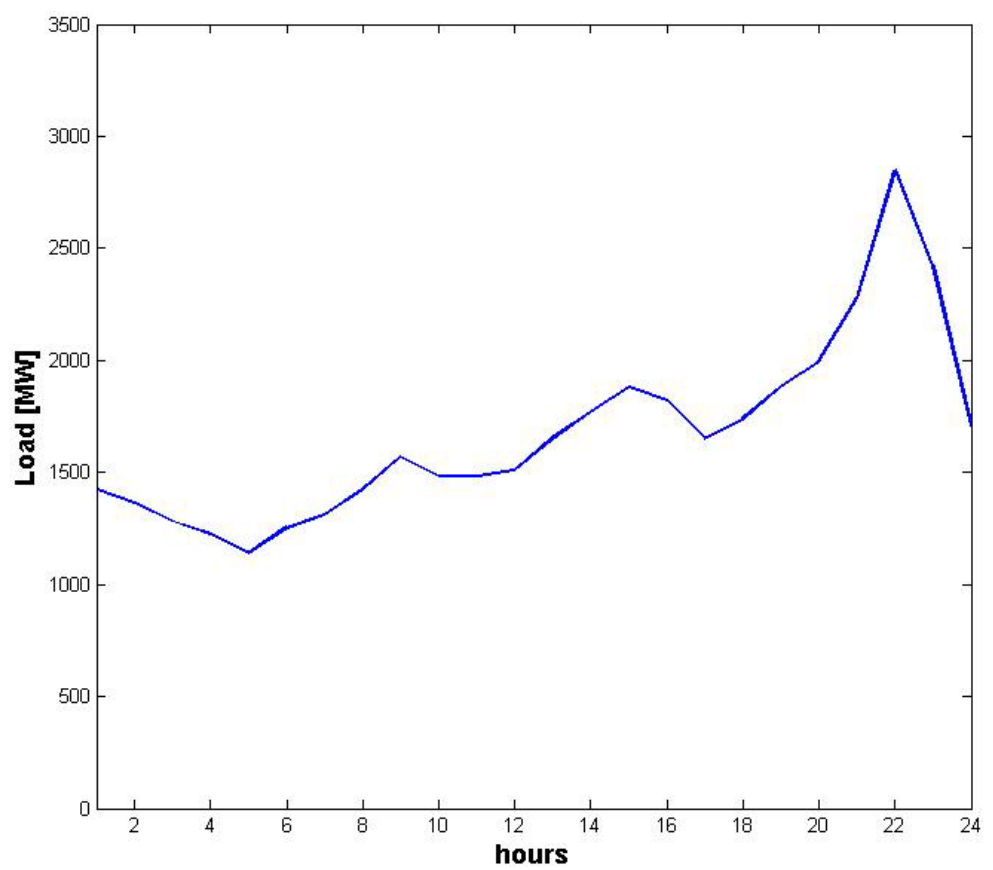

Figure 11. The changed load profile with lower load factor for IEEE 24-bus network

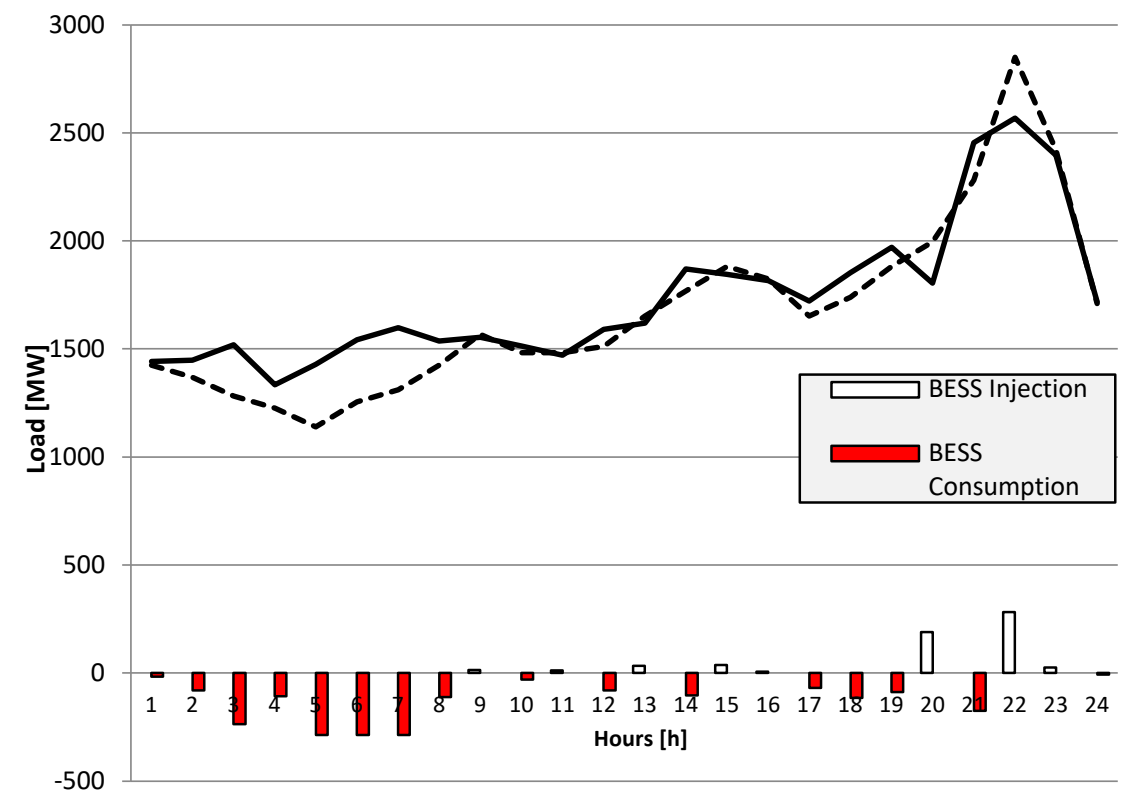

Figure 12. The operating charge/discharge graph of battery storage in IEEE 24-bus grid for case 4 
After implementing the presented load profile in the MO-CSA algorithm, the operation schedule and new load curve could be obtained as presented in figure 12. This plot shows that the proposed storage schedule couldn't improve the load factor as high as the previous one, regarding to the low rate of its energy capacity in comparison with the peak load. But, the improvement of load factor index could be seen and the amount of it reached from 58.67 to 67.53 percent. Besides, the network losses reduced from $2890.6 \mathrm{~kW}$ to $2533.57 \mathrm{~kW}$. In this case, the size of the proposed battery storage is proposed to be $1800 \mathrm{MWh}$ that is installed on bus 22 . Figure 12 presents the daily scheduled operation curve for the proposed storage that helps the network to reach its main objectives.

Figure 12 has clearly shows that the storage facility injects the energy with its maximum power in peak load hours and stores the energy in the base load hours for improving the load factor, network loss and voltage stability index as the main objectives of the problem. The voltage stability index was about 0.8250 in this case. After installation and also operation of the storage, the amount of it decreased and reached to 0.2094. It shows a considerable improvement in the network voltage index. Figure 13 shows the voltage profile of the network in peak load time of the power system.

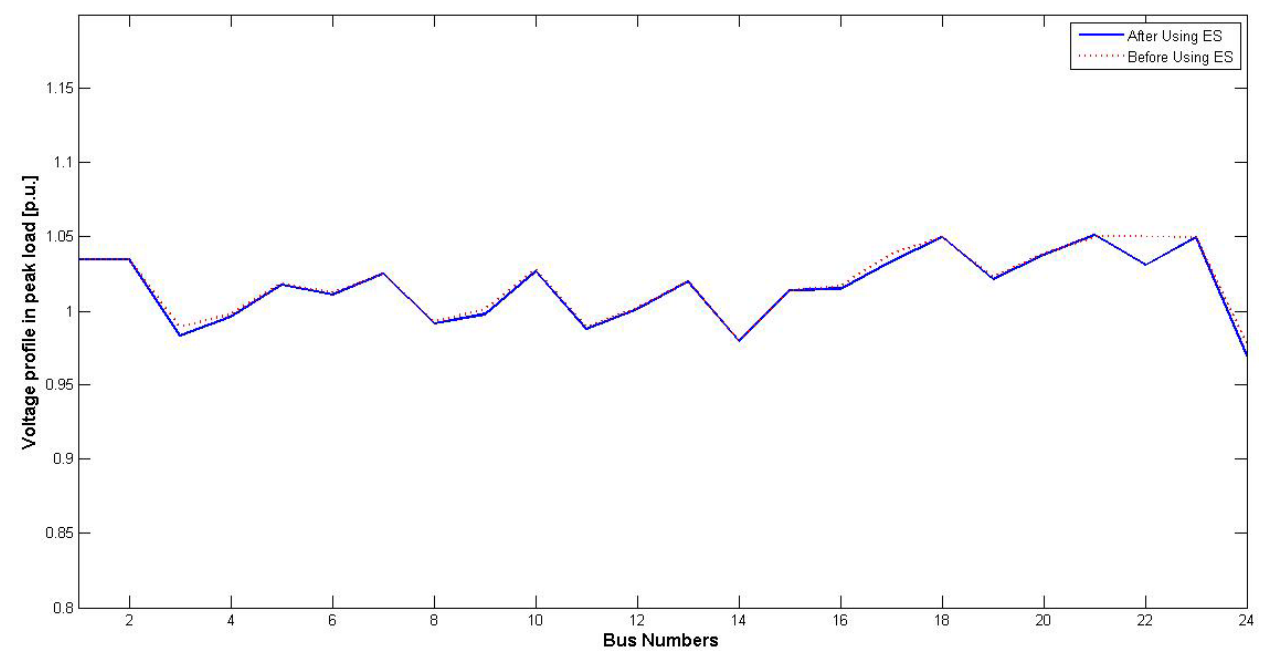

Figure 13. The peak hour voltage profile of IEEE 24-bus network for the case 4

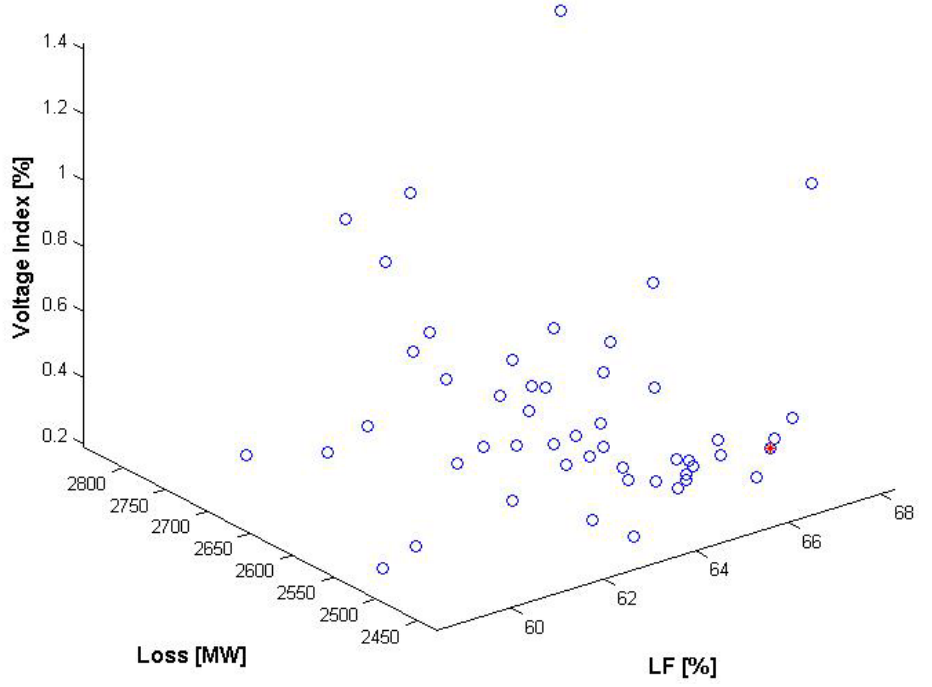

Figure 14. 3D Pareto space for case 4 
Considering the fact that the MO-CSA algorithm in the fourth case study has three objectives, the pareto space should have 3D dimension and could be presented in figure 14. It shows that the pareto front chose the proper answer market by ' $*$ ' sign among the existent results in spite of the fact that there are some other answers with better results in one or two objectives. For more clearing the subject, figure 15 shows the pareto space of purposed algorithm in 2-dimensions for each two pair of objectives. Th $2 \mathrm{D}$ results show that there are optimized responses in some objectives that did not be dominated to be proposed. The presented figures show good diversity of the solutions that have taken from the simulations.
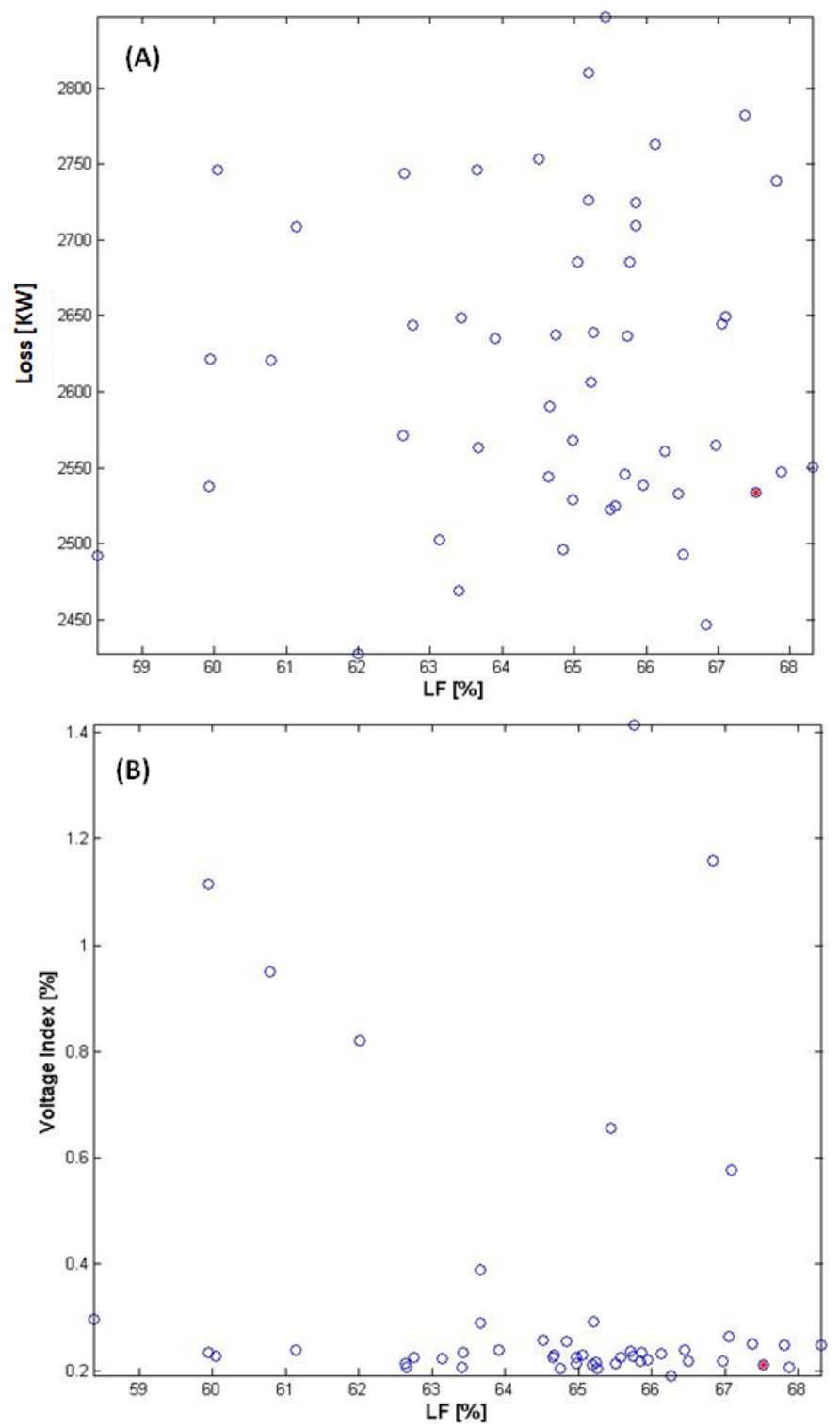

Figure 15. 2D Pareto space for the first case study, load factor ratio versus network losses (A) and network voltage index (B)

Figure 16 shows the proposed position of the storage facility in different case studies for 24bus IEEE network. It shows that the algorithm proposes bus 22 or 6 for installation of battery storage, in most of the cases. Besides, table 1 presents a total conclusion for the achieved results. 


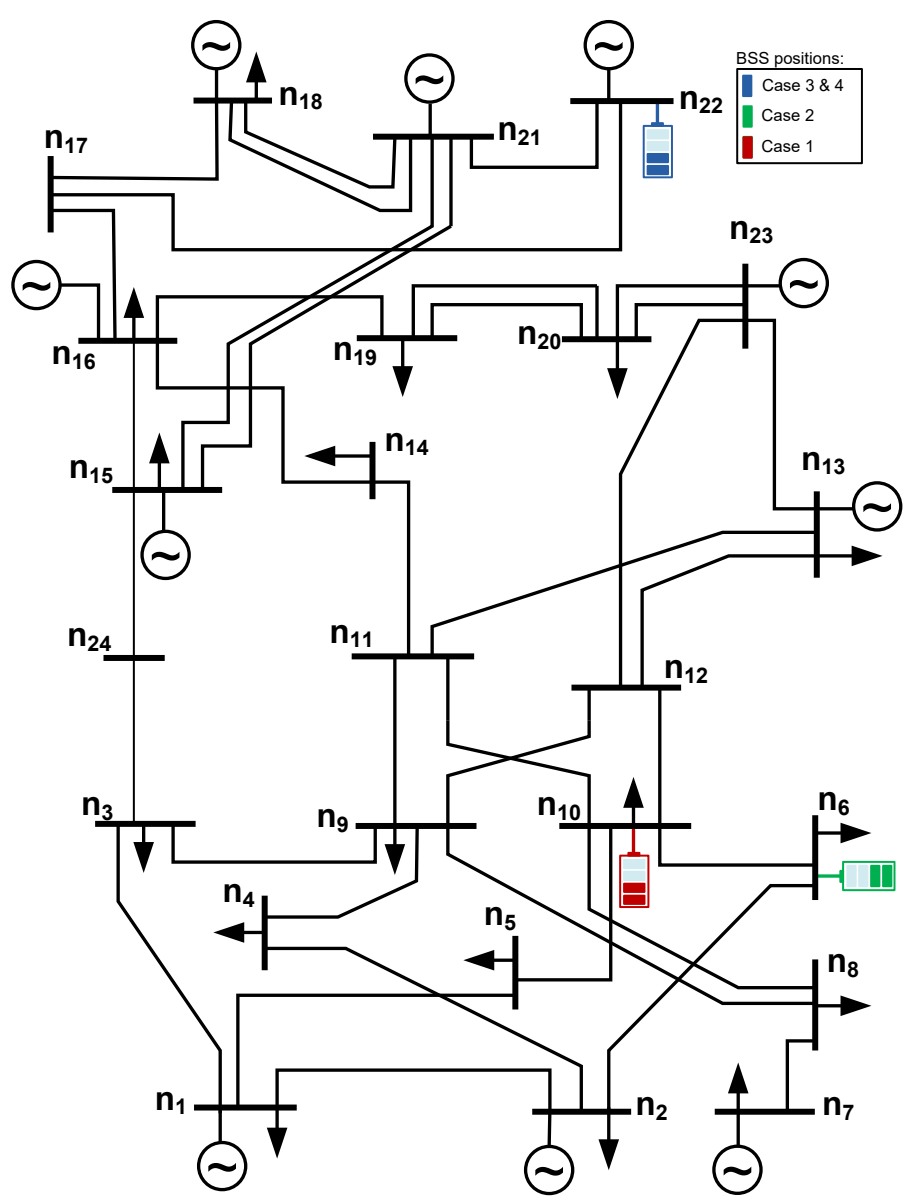

Figure 16. The schematic diagram of IEEE 24-bus network and the optimal places for BESS

In order to accurately evaluate the results of the multi-objective cuckoo search algorithm, another developed algorithm is used to validate the decisions. For this purpose, a multi-objective genetic algorithm function (MOGA) in MATLAB [47], was used for optimization. This function invoked the genetic algorithm for a multi-objective optimization and the global Pareto-optimal solutions were obtained through an MOGA, in this way. The MOGA function gamultiobj implements a controlled elitist genetic algorithm and can be used to solve the multi-objective optimization problem in several variables (A variant of NSGA-II [48]). The gamultiobj can strike the region near an optimal Pareto front relatively quickly, but it can take many function evaluations to achieve remarkable convergence. A non-dominated rank is assigned to each individual using the relative fitness. Several cases were simulated to select proper parameters operated in the genetic algorithm. The following parameters were properly set for the simulation: population size: 100 , crossover fraction: 0.7 , Pareto-front population fraction: 0.8 , generations: 600 and function tolerance: 1E-6. The Pareto front population fraction kept the fittest population down to the specified fraction to maintain a diverse population. This exercise aimed accurately to confirm the operational capability and satisfactory reliability of the previous multi-objective optimization algorithm.

The objective function outcomes from MOGA function, are displayed in the last column of Table 1 for the new load profile. These values were then compared with the objective function values obtained from the MO-CSA optimization. These findings show the CSA algorithm has provided better results in all cases except in storage battery size. Regarding the capacity of the storage, it should be noted that there is not much difference with the results obtained from the 
previous algorithm. Besides, according to other results of this evaluation, the results of MO-CSA seem more acceptable.

Table 1. CSA output results for different case studies

\begin{tabular}{|l|c|c|c|c|c|c|}
\cline { 2 - 7 } \multicolumn{1}{c|}{} & \multicolumn{4}{c|}{ Old Load Profile } & \multicolumn{2}{c|}{ New Load Profile } \\
\cline { 2 - 7 } \multicolumn{1}{c|}{} & Base case & Case 1 & Case 2 & Case 3 & MO-CSA & MOGA \\
\hline $\begin{array}{l}\text { Proposed capacity } \\
\text { [MWh] }\end{array}$ & - & 1800 & 1800 & 1800 & 1800 & 1722 \\
\hline Proposed Bus no. & - & 10 & 6 & 22 & 22 & 10 \\
\hline $\begin{array}{l}\text { Active Power Loss } \\
{[\text { KW] }}\end{array}$ & 1707 & 1665 & 1738 & 1434 & $\begin{array}{c}2534 \\
(2890)\end{array}$ & $\begin{array}{c}2768 \\
(2890)\end{array}$ \\
\hline Loss reduction [\%] & - & 2.5 & -1.8 & 16 & 12.32 & 4.22 \\
\hline $\begin{array}{l}\text { Voltage Stability Index } \\
\text { (VSI) }\end{array}$ & 0.1536 & 0.1416 & 0.1349 & 0.1643 & $\begin{array}{c}0.2094 \\
(0.8250)\end{array}$ & $\begin{array}{c}0.2638 \\
(0.8250)\end{array}$ \\
\hline VSI improvement [\%] & - & 7.8 & 12.17 & -7 & 74 & 68.02 \\
\hline Load Factor [\%] & 82.83 & 88.11 & 87.33 & 88.39 & $\begin{array}{c}67.53 \\
(58.66)\end{array}$ & $\begin{array}{c}65.69 \\
(58.66)\end{array}$ \\
\hline
\end{tabular}

\section{Conclusion}

In this paper, an algorithm is proposed for accurately determining the capacity and position of the required battery storage in a micro-grid. The required battery storage is determined by minimizing the network power factor, system losses and voltage stability index. The presented results show that by increasing the storage capacity of the grid, the important network parameters can obtain considerable improvements. In addition, the results show that if the investment cost of storage facilities was not considered, the system operator typically wants to gain the best usage from all available capacity of the storage. In fact, the optimal size of the storage battery should be a trade-off between the investment cost and improved network parameters. But, considering high prices of the battery storage installation, it seems impossible at this time. But, regarding the severe downfall of the investment cost of them on past years, maybe in the near future we can achieve such approach.

\section{References}

[1]. Luo, X., et al., Overview of current development in electrical energy storage technologies and the application potential in power system operation. Applied Energy, 2015. 137: p. 511536.

[2]. Nykvist, B. and M. Nilsson, Rapidly falling costs of battery packs for electric vehicles. Nature Climate Change, 2015. 5(4): p. 329-332.

[3]. Krim, Y., S. Krim, and M.F. Mimouni, Control and management Strategy of a Generation System Made By Variable-Speed Wind Turbine and Super-Capacitor Energy Storage System for Standalone Applications. International Journal on Electrical Engineering \& Informatics, 2017.9(3).

[4]. Tabasi, M. and P. Asgharian, Optimal Operation of Energy Storage Units in Distributed System Using Social Spider Optimization Algorithm. International Journal on Electrical Engineering \& Informatics, 2019. 11(3).

[5]. Chen, S., H.B. Gooi, and M. Wang, Sizing of energy storage for microgrids. IEEE Transactions on Smart Grid, 2012. 3(1): p. 142-151.

[6]. Ahadi, A., S.-K. Kang, and J.-H. Lee, A novel approach for optimal combinations of wind, PV, and energy storage system in diesel-free isolated communities. Applied Energy, 2016. 170: p. 101-115.

[7]. Vilathgamuwa, D.M., P.C. Loh, and Y. Li, Protection of microgrids during utility voltage sags. IEEE Transactions on Industrial Electronics, 2006. 53(5): p. 1427-1436.

[8]. Wang, Z., et al. A multi-period optimal power flow model including battery energy storage. in Power and Energy Society General Meeting (PES), 2013 IEEE. 2013. IEEE. 
[9]. Nakayama, K., et al. Distributed real-time power flow control with renewable integration. in Smart Grid Communications (SmartGridComm), 2013 IEEE International Conference on. 2013. IEEE.

[10]. Kabir, M., et al., Improving voltage profile of residential distribution systems using rooftop PVs and Battery Energy Storage systems. Applied energy, 2014. 134: p. 290-300.

[11]. Wade, N.S., et al., Evaluating the benefits of an electrical energy storage system in a future smart grid. Energy policy, 2010. 38(11): p. 7180-7188.

[12]. Liu, Y., K. Wu, and D. Zhao. Home energy management optimizing models for residential demand response considering compound des. in Power System Technology (POWERCON), 2014 International Conference on. 2014. IEEE.

[13]. Madlener, R. and J. Latz, Economics of centralized and decentralized compressed air energy storage for enhanced grid integration of wind power. Applied Energy, 2013. 101: p. 299-309.

[14]. Le, H.T. and T.Q. Nguyen. Sizing energy storage systems for wind power firming: An analytical approach and a cost-benefit analysis. in Power and Energy Society General Meeting-Conversion and Delivery of Electrical Energy in the 21st Century, 2008 IEEE. 2008. IEEE.

[15]. Korpaas, M., A.T. Holen, and R. Hildrum, Operation and sizing of energy storage for wind power plants in a market system. International Journal of Electrical Power \& Energy Systems, 2003. 25(8): p. 599-606.

[16]. Oudalov, A., R. Cherkaoui, and A. Beguin. Sizing and optimal operation of battery energy storage system for peak shaving application. in Power Tech, 2007 IEEE Lausanne. 2007. IEEE.

[17]. Kim, H., et al., Impact of battery energy storage system operation strategy on power system: An urban railway load case under a time-of-use tariff. Energies, 2017. 10(1): p. 68.

[18]. Taheri, S.S., S.J. Seyed-Shenava, and M. Modiri-Delshad. Transmission network expansion planning under wind farm uncertainties using cuckoo search algorithm. in Clean Energy and Technology (CEAT) 2014, 3rd IET International Conference on. 2014. IET.

[19]. Bui, L.T., Multi-Objective Optimization in Computational Intelligence: Theory and Practice 2008: IGI Global.

[20]. Modiri-Delshad, M., et al. Optimal Generation Scheduling in Microgrids by Cuckoo Search Algorithm. in Clean Energy and Technology (CEAT) 2014, 3rd IET International Conference on. 2014. IET.

[21]. Moradi, M.H., et al., An efficient hybrid method for solving the optimal sitting and sizing problem of DG and shunt capacitor banks simultaneously based on imperialist competitive algorithm and genetic algorithm. International Journal of Electrical Power \& Energy Systems, 2014. 54: p. 101-111.

[22]. Pawelek, R., et al. Study on operation of energy storage in electrical power microgridModeling and simulation. in Harmonics and Quality of Power (ICHQP), 2010 14th International Conference on. 2010. IEEE.

[23]. Taheri, S.S., J. Kazempour, and S. Seyedshenava, Transmission expansion in an oligopoly considering generation investment equilibrium. Energy Economics, 2017. 64: p. 55-62.

[24]. Taheri, S.S. and S.-J. Seyedshenava, Strategic bidding of a GENCO in multi-period electricity auction: a bi-level approach. International Journal of Mathematical Modelling and Numerical Optimisation, 2016. 7(3-4): p. 293-306.

[25]. Yang, X.-S. and S. Deb. Cuckoo search via Lévy flights. in Nature \& Biologically Inspired Computing, 2009. NaBIC 2009. World Congress on. 2009. IEEE.

[26]. Yang, X.-S. and S. Deb, Engineering optimisation by cuckoo search. International Journal of Mathematical Modelling and Numerical Optimisation, 2010. 1(4): p. 330-343.

[27]. Banaei, M.R., B. Mohammadzadeh, and R.R. Ahrabi, Damping of low frequency electromechanical oscillations using UPFC based on cuckoo optimization algorithm. International Journal on Electrical Engineering and Informatics, 2014. 6(4): p. 769. 
[28]. Muhammad, R.D., et al., Modal Analysis and Stability Enhancement of 150 kV Sulselrabar Electrical System using PSS and RFB based on Cuckoo Search Algorithm. International Journal on Electrical Engineering and Informatics, 2017. 9(4): p. 800-812.

[29]. Nguyen, T.T., D.N. Vo, and B.H. Dinh, Cuckoo search algorithm using different distributions for short-term hydrothermal scheduling with reservoir volume constraint. International Journal on Electrical Engineering and Informatics, 2016. 8(1): p. 76.

[30]. Brown, C.T., L.S. Liebovitch, and R. Glendon, Lévy flights in Dobe Ju/'hoansi foraging patterns. Human Ecology, 2007. 35(1): p. 129-138.

[31]. Reynolds, A.M. and M.A. Frye, Free-flight odor tracking in Drosophila is consistent with an optimal intermittent scale-free search. PloS one, 2007. 2(4): p. e354.

[32]. Vadivelu, K. and G.V. Marutheeswar, Multi Objective Optimization based optimal Reactive Power Planning Using Improved Differential Evolution Incorporating FACTS. International Journal on Electrical Engineering and Informatics, 2015. 7(4): p. 630.

[33]. Hota, P.K., N.C. Sahu, and A.K. Barisal, Multi Objective Generation Dispatch of a Hybrid System with Fuzzy based Space Search Algorithm. International Journal on Electrical Engineering \& Informatics, 2016. 8(3).

[34]. Shi, Q. and J. JaJa, A new framework for addressing temporal range queries and some preliminary results. Theoretical computer science, 2005. 332(1-3): p. 109-121.

[35]. Gembicki, F. and Y. Haimes, Approach to performance and sensitivity multiobjective optimization: The goal attainment method. IEEE Transactions on Automatic control, 1975. 20(6): p. 769-771.

[36]. Niknam, T., A new fuzzy adaptive hybrid particle swarm optimization algorithm for nonlinear, non-smooth and non-convex economic dispatch problem. Applied Energy, 2010. 87(1): p. 327-339.

[37]. Daneshi, H., et al. Mixed integer programming method to solve security constrained unit commitment with restricted operating zone limits. in 2008 IEEE International Conference on Electro/Information Technology. 2008. IEEE.

[38]. Walters, D.C. and G.B. Sheble, Genetic algorithm solution of economic dispatch with valve point loading. IEEE transactions on Power Systems, 1993. 8(3): p. 1325-1332.

[39]. Basu, M., A simulated annealing-based goal-attainment method for economic emission load dispatch of fixed head hydrothermal power systems. International Journal of Electrical Power \& Energy Systems, 2005. 27(2): p. 147-153.

[40]. Amjady, N. and H. Nasiri-Rad, Nonconvex economic dispatch with AC constraints by a new real coded genetic algorithm. IEEE Transactions on Power Systems, 2009. 24(3): p. 1489-1502.

[41]. He, X.-s., N. Li, and X.-S. Yang. Non-dominated sorting cuckoo search for multiobjective optimization. in Swarm Intelligence (SIS), 2014 IEEE Symposium on. 2014. IEEE.

[42]. Yang, X.-S. and S. Deb, Multiobjective cuckoo search for design optimization. Computers \& Operations Research, 2013. 40(6): p. 1616-1624.

[43]. Yang, X.-S., Nature-inspired metaheuristic algorithms. 2010: Luniver press.

[44]. Gandomi, A.H., X.-S. Yang, and A.H. Alavi, Cuckoo search algorithm: a metaheuristic approach to solve structural optimization problems. Engineering with computers, 2013. 29(1): p. 17-35.

[45]. Grigg, C., et al., The IEEE reliability test system-1996. A report prepared by the reliability test system task force of the application of probability methods subcommittee. IEEE Transactions on power systems, 1999. 14(3): p. 1010-1020.

[46]. Conejo, A.J., M. Carrión, and J.M. Morales, Decision making under uncertainty in electricity markets. Vol. 1. 2010: Springer.

[47]. MATLAB The Language of Technical Computing. 2017, Math Works (2000), Inc.: MA, USA.

[48]. Deb, K., et al., A fast and elitist multiobjective genetic algorithm: NSGA-II. IEEE transactions on evolutionary computation, 2002. 6(2): p. 182-197. 


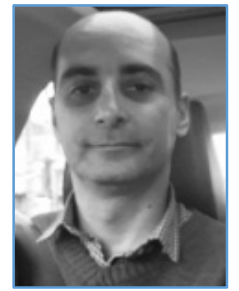

S. Saeid Taheri received his B.Sc. and M.Sc. degrees in electrical engineering from Shahid Beheshti University and Tehran Polytechnic, Iran, respectively, and Ph.D. Degree from University of Mohaghegh Ardabili in 2017. Currently, he is an Assistant Professor at Islamic Azad University, Sarab Branch, Iran. His research interests mainly include the study of mathematical or heuristic modeling which is related to electrical problems within different environments using multi-level and multi-objective optimization models, during these years.

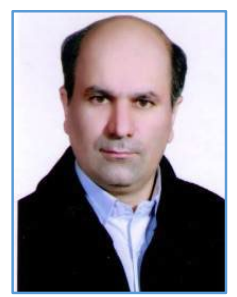

Seyed-Jalal Seyed-Shenava received the B.Sc. degree in Electrical Engineering in 1991 from Tehran University, Tehran, Iran, and the M.Sc. and Ph.D. degree in Electrical Power Engineering from Tarbiat Modares University, Tehran, Iran, in 1994 and 2008, respectively. He is an Associate Professor in University of Mohaghegh Ardabili, Ardabil, Iran. His research interests include electrical distribution and power system planning and operation.

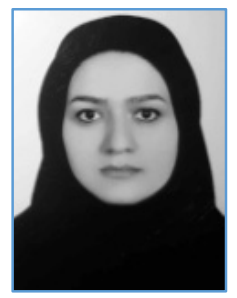

Vahideh Mohadesi received the B.Sc. degree in Physics from Tehran University, Iran, and the M.Sc. and Ph.D. degree in Photonics from University of Tabriz, Iran, in 2018. She is an Assistant Professor at Islamic Azad University, Sarab Branch, Iran. Her research interests include the study of modeling electronic and photonic devices and using optimization techniques to improve their performance, during these years.

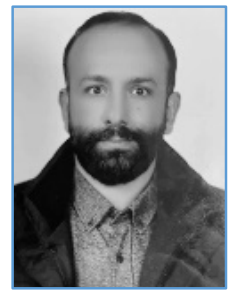

Rasoul Esmaeilzadeh was born in Tabriz, Iran, in 1978. He received the M.Sc. degree in Electrical Engineering - Control in 2003 from University of Tabriz and Ph.D. degree in Electrical Engineering-Power from Shahid Madani University, Tabriz, Iran in 2018. Currently, he is the Manager of R\&D department of Azerbaijan Regional Electric Company. His research interests are the application of control theories and optimization algorithms in power system, dynamic load modeling and energy bidding. 